\title{
Premio Nacional de Medicina 2020: Dr. Vicente Valdivieso Dávila
}

\author{
José Luis Chianale B. ${ }^{1}$
}

\author{
National Medicine Award 2020: Dr. Vicente Valdivieso Dávila
}

El Premio Nacional de Medicina es un reconocimiento creado en el año 2001 por la Academia Chilena de Medicina, la Asociación de Facultades de Medicina de Chile (ASOFAMECH), la Asociación de Sociedades Médicas de Chile (ASOCIMED) y el Colegio Médico de Chile, con el fin de reconocer la obra de aquellos médicos que han sobresalido entre sus pares por su exitosa trayectoria en el área clínica o de salud pública y que, además, han tenido un rol destacado en la docencia, administración académica o la investigación. Este reconocimiento se otorga cada dos años y en la décima edición del premio más importante que se entrega en Chile a los médicos, se distinguió al médico internista de la Pontificia Universidad Católica de Chile y especializado en gastroenterología en la Universidad de California (UCLA) Dr. Vicente Valdivieso Dávila.

El Dr. Valdivieso se incorpora a la Sociedad Chilena de Gastroenterología en el año 1968, atraído por su amigo e investigador Dr. Héctor Orrego, y preside la Sociedad Chilena de Gastroenterología entre los años 1976 y 1978. Fue un activo impulsor del Departamento de Gastroenterología y del desarrollo de la Escuela de Medicina de la Pontificia Universidad Católica de Chile, siendo su Director entre los años 1980 a 1982; ocupó diversos cargos de gestión académica en la Escuela y la Universidad, culminando su carrera académica como Profesor Titular. Desarrolló una productiva línea de investigación en torno a la patogenia de la colelitiasis y el metabolismo de los lípidos biliares, con más de 50 publicaciones nacionales e internacionales; en un período donde la curiosidad científica se volcaba hacia los mecanismos de las enfermedades. Como señala él mismo en una entrevista años atrás, marcado por su experiencia en la UCLA: "Se discutía a fondo por qué razón comenzaba una enfermedad y por qué razón evolucionaba de una manera u otra". Su curiosidad por los mecanismos fisiopatológicos y celulares de las enfermedades lo ha acompañado toda su vida y ha marcado a varias generaciones de médicos que se formaron bajo su influencia a lo largo de estas décadas. El por qué de los mecanismos se proyecta también a otros ámbitos de la vida, más allá de la medicina, cubriendo sus reflexiones con el manto del rigor en el uso de la razón, probablemente una de sus características más destacadas y notables.

Realizó numerosas contribuciones en distintas instituciones académicas en las que participó muy activamente. Es miembro de número de la Academia de Medicina de Chile, fue Presidente de la Sociedad Médica de Santiago, fue Presidente de la Corporación Nacional de Certificaciones Médicas (CONACEM) por más de 15 años y fue Secretario Ejecutivo de la Agencia de Acreditación de los Programas Universitarios de Especialidades Médicas (APICE), testimonios patentes de su profunda vocación de servicio, fundamentalmente orientada a la formación de especialistas de calidad para el país, una de sus más profundas preocupaciones en el devenir de la medicina en Chile. Ha contribuído al programa AUGE en patologías gastrointestinales y fue un gran impulsor del concurso de proyectos de investigación y desarrollo en salud, Fonis.

Ha recibido, por sus destacadas contribuciones y su dilatada trayectoria, numerosos reconocimientos a lo largo de su carrera, destacando entre ellos Profesor Emérito de la Pontificia Universidad Católica de Chile, Maestro de la Medicina Interna Chilena y el año 2020 el máximo galardón que otorgan los pares a un médico: Premio Nacional de Medicina.

Para quienes hemos tenido el privilegio de conocerlo durante estos años, creemos que el Dr. Vicente Valdivieso Dávila representa el mejor testimonio nacional del rigor académico al servicio de la formación médica. ¡Gracias, gracias por todo Dr. Valdivieso!

\author{
Departamento \\ Gastroenterología \\ Pontificia Universidad \\ Católica de Chile, \\ Santiago, Chile \\ Recibido: 5 de mayo \\ de 2021 \\ Aceptado: 10 de \\ mayo de 2021 \\ Correspondencia a: \\ Dr. José Luis Chianale \\ Profesor titular \\ Departamento de \\ Gastroenterología \\ Facultad de Medicina \\ Pontificia Universidad \\ Católica de Chile \\ Diagonal Paraguay \\ 362, 4 piso. Santiago, \\ chile.chianale@
} gmail.com 